\title{
SIETE RAZONES POR LAS QUE VALE LA PENA INVESTIGAR EN MEDICINA EN COLOMBIA
}

\author{
RanNiery Acuña Cordero \\ ${ }^{1}$ Oficial Fuerza Aérea de Colombia, Pediatra Neumólogo, Epidemiólogo Clínico, \\ Especialista en Docencia Universitaria. Grupo de Investigación Respira Latino-América. \\ Subdirector de Docencia e Investigación Hospital Militar Central. \\ Correspondencia: ranniery.acuna@hospitalmilitar.gov.co, ranyacuna@hotmail.com
}

Recibido: Abril 15 de 2014 Aceptado: Junio 9 de 2014

\section{Introducción}

De manera cotidiana siempre se ha considerado que el fin último de la investigación médica en cualquier país debe tener como propósito único la generación y apropiación de conocimiento necesarios para garantizar el mejor estado de salud posible sobre la mayor cantidad de pobladores que le constituyen. Se trata entonces, de una transferencia de conocimiento encaminado a utilizar la mejor evidencia disponible para ofrecer un tipo de atención médica racional e integral, y realmente bajo la óptica de una concepción filantrópica, es indudable que en cualquier latitud debe ser así. Colombia intenta no alejarse de este mismo objetivo, no sólo en cuanto a que su Constitución Política (Capítulo II, Artículos 44 y 49, Constitución Política de Colombia, 1991) consigna que dentro de los derechos fundamentales que le acompañan a cada uno de sus ciudadanos y que son directa responsabilidad del estado ofrecerlos, están aquellos relacionados con el derecho a la seguridad social en salud y la vida, aspecto este último de especial relevancia especialmente en población infantil, articulado éste que de cumplirse a cabalidad garantizaría el apropiado funcionamiento y bienestar de su economía, al disponer de una población laboralmente activa inmersa dentro de las mejores condiciones e indicadores de salubridad posibles. Así mismo, la investigación médica desarrollada sobre su propia comunidad, como también ocurre con cualquier otra nación a nivel global, le permite obtener el asidero ideal para operar a partir de la toma de decisiones, con la mejor asertividad respecto al manejo de la salud con base en los principios de calidad y costo efectividad. En palabras sencillas, se trata de administrar de la mejor manera los muy escasos recursos asignados para las estrategias de salud sobre el mayor número de beneficiarios posible y ojalá invertidos especialmente en planes de promoción y prevención. Ningún país en el mundo es ajeno a estos mismos principios de austeridad y de eficiencia para la inversión de sus escasos recursos sanitarios, y de manera inequívoca, todos convergen en el concepto de la investigación como una herramienta fundamental a la hora de encontrar soluciones eficaces. En ese orden de ideas la investigación médica, si resulta ética y bien estructurada, modifica favorablemente los aspectos más relevantes de la sociedad, entre los que se involucran sus hábitos, idiosincrasias, modus vivendi y expectativa de vida (Lewinson, 2008).

Más recientemente la investigación clínica ha pretendido hacer hincapié en la evaluación de la calidad de vida no solo de los sujetos que son motivo de la observación sino además se extiende en su análisis hasta la persona o grupos de personas sobre quienes recaen la responsabilidad de atender y cuidar a esa misma población afecta, y por ende el grado en que se distorsiona su calidad de vida (Pedraza, Rodríguez y Acuña, 2013) y cuánto le cuesta a un país perder su capacidad laboral. Sin lugar a dudas un conjunto de soluciones bien administradas a todos estos aspectos, y por sobre todo, bien aplicadas pueden dejar muy bien parada la gestión administrativa de un país 
y la calidad de vida de sus habitantes. Resulta curioso sino coincidencial que al unísono de los derechos fundamentales consignados en la carta magna también están el derecho fundamental "a la enseñanza, aprendizaje, investigación y cátedra" (Capítulo II, Artículos 64-67, Constitución Política de Colombia 1991). Visto desde la perspectiva puramente teórica, los gobernantes de un país o al menos el de Colombia, a la luz de la jurisprudencia que acompaña su constitución, debería garantizar en todo caso que en el ejercicio de las profesiones sin exclusión, existiera la aplicación de estas cuatro disciplinas en forma continua y universal.

Resulta imposible no reconocer el esfuerzo que durante el último quinquenio el Gobierno de Colombia ha dedicado al mejoramiento, sin endilgar ínfulas de victoria apabullante, en la modernización de la enseñanza y el aprendizaje, y sincronizado con Tuning Latinoamerica (2007) sus esfuerzos resultan poco a poco evidentes en la transición educativa desde la obsoleta metodología tradicional (ebria de positivismo) y centrada desquisiadamente en contenidos hacia una metodología mucho más coherente, participativa y reflexiva con fundamento en la adquisición de competencias en donde su esencia tiene mucho que ver epistemológicamente con el constructivismo, preferiblemente social, y donde el alumno, sin importar su disciplina, estructura su enfoque a partir del conocimiento anidado en una perspectiva de construcción y de renovación permanente (Pinilla, 2011).

Infortunadamente la investigación sigue siendo la cenicienta de la historia. Los observatorios internacionales de Ciencia y Tecnología, el Banco Mundial y diferentes agencias económicas como América Economía han reconocido en la investigación uno de los indicadores más relevantes a la hora de cuantificar el nivel de desarrollo de un país. En sus apreciaciones han conceptuado que para los escenarios más modestos al menos el 1\% del PIB de la nación deben ser reinvertidos en propósitos de investigación. Es así como el Banco Mundial, metódicamente y en forma bianual, a través de su oficina del Instituto de Estadística de la Organización de las Naciones Unidas para la Educación, la Ciencia y la Cultura (UNESCO, 2012), concluye qué países realmente manejan políticas para la investigación y cuando se repara en sus indicadores se puede concluir fácilmente que los países mejor rankeados en términos de desarrollo y mayor productividad científica, son precisamente aquellos en los que se asigna al menos el $1 \%$ del PIB para programas de investigación. Colombia asigna apenas un $0,16 \%$ de su PIB para estos menesteres. Es así como naciones consideradas "del primer mundo" como Alemania asignan hasta un 2,82\%, Estados Unidos 2,79\%o Israel, que encabeza el listado, con un impresionante 4,27\% de su PIB. Resulta desalentador reconocer que incluso países como Ecuador $(0,26 \%)$ y Costa Rica $(0,40 \%)$ con economías mucho menos sólidas que la nuestra, invierten un mayor porcentaje de su PIB para el financiamiento de investigación e innovación (Banco Mundial, data 2013).

Un caso sorprendente en la región es el de Brasil, país que desde el año 2008 mantiene su inversión por encima de 1\% del PIB para programas de Investigación y Desarrollo, y que gracias a esta medida, logró doblar, en tan sólo un quinquenio, su producción científica, pasando de 23.962 publicaciones en 2005, cuando invertía solo el 0.57\% de su PIB a 49.664 publicaciones en 2011 (1.08\% de su PIB). Del total de las publicaciones anteriormente citadas, 5.881 correspondieron al campo de la medicina en el año 2005 y 13.122 en el año 2011. En cifras globales, Brasil contribuyó en 2011 al 2,12\% del total de la producción científica mundial y fue responsable del 54\% de la investigación científica a nivel regional. Muy diferente es el caso de Colombia que continua obstinada en la asignación de apenas un 0,16 \% del PIB en los 2 últimos años alcanzando una productividad científica general de 1.387 publicaciones en 2005 y de 4.708 en el año 2011, con respecto al aérea de la medicina, 348 y 1.092 fueron las publicaciones prensadas respectivamente. El resumen de los datos generales muestran que Colombia paupérrimamente contribuye con tan solo el $0.20 \%$ de toda la producción científica mundial y con el $5.2 \%$ de la regional. No hay que ser necesariamente un estadístico para argüir que nuestra investigación resulta minúscula para la realidad científica mundial. A pesar de ello Colombia para el año 2011, consiguió ubicarse en el puesto 48 entre 226 naciones discriminando únicamente por publicaciones médicas, totalizando 1.092 (aunque indizadas solo 997) y ubicándose incluso por encima de Cuba que prensó durante ese mismo año 850 (solo indizadas 804). Para este tipo de clasificaciones, la proporción de inversión 
del PIB se relacionó en forma directamente proporcional con el puesto alcanzado en términos de productividad médica científica, ubicándose por encima nuestro Brasil, en el puesto 13, Argentina en el 37 y Chile en el 44. (SCI mago Journal and Country Rank, 2013)

\section{Razones por las cuales "Si" vale la pena investigar sobre medicina en Colombia}

\author{
Razón \#1. Economías emergentes, oportunidades de \\ investigación emergentes
}

Por su actual situación económica Colombia hace parte de la categoría de países emergentes rotulados como de ingreso mediano alto. El PIB de 333,4 mil millones reportado para el año 2011, una economía con comportamiento muy optimista, creciendo sostenidamente por encima de un $4 \%$ durante los últimos 5 años, una reducción en la tasa de incidencia de la pobreza de casi el 10\% en los 6 años anteriores (34\% viven ahora por debajo de la línea de pobreza en comparación con $45 \%$ en 2005), niveles de inestabilidad social tolerables, un notable avance, aunque todavía insuficiente, en la profesionalización de sus investigadores y por sobretodo con una clara política abierta a la inversión extranjera, son factores que se constituyen relevantes para que los organismos internacionales de salud y la industria farmacéutica mundial ponga sus ojos y apueste a la siembra de propuestas de investigación en nuestro país.

Esta tendencia de desarrollar investigación en países de bajos y medianos ingresos, actualmente tiene una vigencia global. Entre otras, por la escasa validez externa que aportan los ensayos clínicos controlados en países desarrollados en los cuales su metodología casi siempre demanda la pesquisa de una población extremadamente homogénea, por no decir casi perfecta. Colombia con casi 48 millones de habitantes, distribuidos a lo largo 6 subregiones geopolíticas, con multiplicidad de razas y mestizajes, diversidad en clases sociales, niveles diferentes de educación e idiosincrasias y amén de compartir algunas costumbres arraigadas en otros países que componen la civilización occidental, ofertan una importante condición de validez interna y externa en su población muestra. Precisamente estas características podría detonar un sinnúmero de investigaciones médicas de tipo pragmático en las cuales la diversidad está a la orden del día, facilitando la observación del comportamiento de terapias y medicamentos en diversos escenarios de vida real y con tamaños de la muestra generosos que podrían superar a muchos países de la región como Chile o incluso Argentina que ocupa el tercer lugar en importancia en investigación médica en Latinoamérica. Adicionalmente la normatividad vigente en cabeza del Invima y supervisada estrictamente en los centros de investigación reconocidos mediante la aplicación de la Buena Práctica Clínica, beneficia particularmente la celeridad en su trámite administrativo al desarrollo de estudios de fase IV. Esto no quiere decir que no haya campo para la apuesta sobre estudios de fase I, II y III. De hecho se ha calculado que los costos de este tipo de estudios, un factor extraordinariamente importante a la hora concebir un protocolo clínico, en países de bajo y mediano ingreso, puede resultar hasta un tercio del valor de lo que el mismo estudio costaría si se corriera en los Estados Unidos (Lang, 2012, Patsoupolus, 2011)

Adicionalmente los países emergentes tienen a su disposición mayores tamaños de la muestra considerados muy útiles y muy diversos a la hora de evaluar si el comportamiento fármaco-cinético de una molécula resulta similar al de sujetos pertenecientes a grupos poblaciones con mejor calidad de vida, y de lejos, lo más importante determinar si los perfiles de seguridad del medicamento o terapia que se está investigando tiene un comportamiento inequívoco y universal en todo tipo de razas, comunidades, en poblaciones con morbilidades asociadas o diferentes escenarios sociales.

No menos relevante para favorecer y fortalecer la investigación, resulta el hecho de que el reclutamiento de sujetos resulta algo más fácil en países como el nuestro, teniendo como base el sentimiento de confianza que aún se percibe acerca del actuar médico y en algunos sentimientos de calidez que, a pesar de la ley 100, sobreviven estoicamente en la relación médico-paciente y es así como no resulta cotidiano en Colombia, realizar reclutamiento de sujetos por intermedio de avisos publicados en la prensa o vía internet, sino a través de la invitación misma del médico tratante. De otro lado, nuestros inquietos investigadores saben que al correr una investigación en un país como el nuestro deberán estar dispuestos a atender hasta el más mínimo requerimiento sin importar que el mismo no esté directamente relacionado con los objetivos del estudio 
mismo, situación que es prácticamente inexistente en la investigación de países del primer mundo.

Otro argumento importante para potencializar la producción científica en el área médica en Colombia radica en el extraordinario potencial que tiene el país para investigar acerca de enfermedades huérfanas o de baja incidencia. La pérdida de interés que detentan, en este tipo de enfermedades, países con economías más sólidas y por sus mejores estándares de salud, con menor incidencia, facilitan los escenarios para buscar respuestas en la efectividad de maniobras de prevención, el mejoramiento en la calidad de vida en aquellos en quienes la enfermedad ya esté presente o incluso, como forma de pensamiento utópico, en la cura de estas enfermedades y su extrapolación, hasta donde la epidemiologia lo permita, de los aciertos logrados localmente sobre sujetos afectados por la misma condición clínica hacia países con baja incidencia de la misma enfermedad.

También el descubrimiento de nuevas estrategias, en algo más costo-efectivas, que se prueben con éxito en países emergentes con respecto a la adherencia a terapias en investigación o a los protocolos mismos, son susceptibles de ser extrapoladas a países desarrollados, aporte nada despreciable si se tiene en cuenta que ni siquiera en escenarios irreales como lo son los experimentos clínicos controlados en donde difícilmente se logra una adherencia del $90 \%$ y ni hablar de la vida real en donde el paciente se adhiere a tratamientos crónicos si acaso a un $40 \%$.

Finalmente el país tiene las condiciones necesarias para liderar un campo hasta ahora poco explorado en el quehacer de la investigación. Desde nuestra perspectiva como epidemiólogos, bien clínicos o bien generales, se nos ha enseñado que la verdad verdadera proviene solamente del tipo de investigación que se anida en el método científico, la misma que advierte diferencias o asociaciones bajo la óptica de la significancia o de la ejecución sistémica de complejos análisis estadísticos. Toda conclusión a la cual le subyace a un método cuantitativo, siempre y cuando en su aplicación técnicamente sea correcto, involucra números. Pero qué hay de aquellos aspectos relacionados, por ejemplo con la idiosincrasia de un país?, sus tenores sociales, sus necesidades y por sobre todo, las soluciones más convenientes a sus conflictos reales? .Es en este punto donde otro tipo de estudios de orden cualitativo, o acaso por qué no mixtos y transdisciplinarios pueden facilitar el perfeccionamiento de soluciones de manera integral y contextualizada a la problemática nacional. Los estudios antropológico- sociales combinados, por ejemplo, con diseños epidemiológicos que infieran causalidad podrían lo- grar una perspectiva menos miope acerca de la injerencia que tiene la sociedad sobre la enfermedad y el individuo mismo. Este tipo de estudios aún no son muy bienvenidos en países con mayor trayectoria en investigación, generalmente porque no lucran, pero en países como el nuestro en donde su sociedad está dispuesta a ser observada y ser escuchada con la esperanza de que alguna vez su encrucijadas sociales sean resueltas, definitivamente se acompañan de mucho potencial.

\section{Razón \# 2. Inclusión de la investigación en políticas gubernamentales}

Actualmente la responsabilidad de la producción científica, no solo de medicina, recae sobre Colciencias. El Gobierno de Colombia ha tratado de impulsar en serio, aunque con muchos tropiezos la investigación, pero con estrategias que no siempre han resultado contundentes. Conscientes de la importancia que denota para un país el impulso de la investigación e innovación en el año 2009 promulgó la Ley 1286 de Ciencia, Innovación y Tecnología la cual elevó la categoría administrativa de Colciencias a departamento administrativo de Ciencia y Tecnología con el propósito de ser una entidad asesora y con línea directa al gobierno nacional. Visto de otro modo, tendría las potestades suficientes para hablar acerca de temas de investigación al oído directo del Presidente. Sin embargo las buenas intenciones en algún momento se enredaron y dejaron irónicamente a Colciencias como "entidad que no tenía cerebro" (Fog, 2.012) con un recorte presupuestal cercano al 30 \% (550 mil millones frente a 388. mil millones) que castraba irremediablemente los objetivos trazados para el año 2013 reduciendo hasta casi un 50\% de sus metas. Esta situación obligó a una profunda reforma estructural al interior de la Institución que actualmente se está implementando.

No obstante, se debe reconocer que no todo ha sido malo $y$ a esta entidad se le atribuye el hecho de que, al menos los investigadores a nivel nacional, estemos reconocidos $y$ organizados en grupos de trabajo. De igual manera ha tenido un papel fundamental en el monitoreo de la indización de revistas científicas que se prensan en el país. Sus dos plataformas ScienTI y Publindex son garantes, cada uno, de estos menesteres, respectivamente. ScienTI a través de Gruplac tiene como objeto referenciar y desglosar las minucias propias a cada grupo de investigación reconocido en el país. En Diciembre de 2.012, 5.510 de 13.727 grupos registrados en la plataforma cumplieron con los criterios para ser reconocidos con base en el nue- 
vo "modelo de medición de grupos de Investigación, Tecnológica o Innovación, año 2011-2012" (Informe Ejecutivo de la convocatoria nacional para la definición de grupos de Investigación, Tecnológica o Innovación Año 2012, Colciencias, Febrero 2013) es decir, poco menos del $50 \%$ de los grupos presentados fueron considerados apropiados para generar, apropiar o transferir conocimiento, porcentaje para nada despreciable de no reconocidos, lo cual habla de un proceso serio en el que no cualquier grupo de fulanos con unas buenas intenciones pero sin la estructura y organización necesarias, pueden sentarse a investigar. Para esta convocatoria (Colciencias, 2012) las condiciones de reconocimiento de grupos se tornaron mucho más estrictas y apalancadas sobre la esencia misma de la investigación, lo cual trajo como resultado una reducción de casi un $1 \%$ con respecto a los grupos reconocidos en el año 2010. De nuevo, este ejercicio refleja la actual disposición que tiene Colciencias de depurar y entregar un nivel más competente a la investigación en Colombia. Las estadísticas también le han resultado muy alentadoras al estímulo para la creación de nuevos grupos de investigación. Si comparamos los grupos reconocidos hace 10 años frente a los del año 2012 observamos que ha habido un incremento, para nada despreciable, casi 10 veces mayor (544 grupos vs 5.510 grupos, respectivamente) con respecto a los reconocidos en 2002. La lectura que le podemos dar a este crecimiento se explica no solo en el buen trabajo que puede estar haciendo Colciencias en términos de fomentar estímulos para la investigación sino además en la construcción progresiva de un hábito investigativo que antes no existía en el país y quizás a la formación, con mejores competencias, de nuevos investigadores durante la última década, cuyo interés ha sido el de encontrar soluciones propias a los enigmas de salubridad locales. (Ver Figura 1.)

Adicionalmente, los presupuestos asignados para investigación, aunque escasos-en términos de investigación médica ningún presupuesto resulta suficiente para ningún país- también han venido en incremento desde una cifra asignada de 84,3 mil millones en el año 2000 hasta alcanzar los 270 mil millones en el 2013. Estos esfuerzos han repercutido notoriamente en el interés por la creación de nuevos grupos y nuevas líneas de investigación inscribiéndose 13.727 en el año 2012 frente a 1.522 en el 2002 (incremento del 88\%). La promesa que ha hecho el presidente de la República en cabeza del Ministerio de Hacienda de invertir hasta el $10 \%$ de las regalías del país en el fomento y financiación a los modelos de investigación, desarrollo e innovación se constituyen en buenas noticias y en un estímulo adicional para decidirse a investigar.

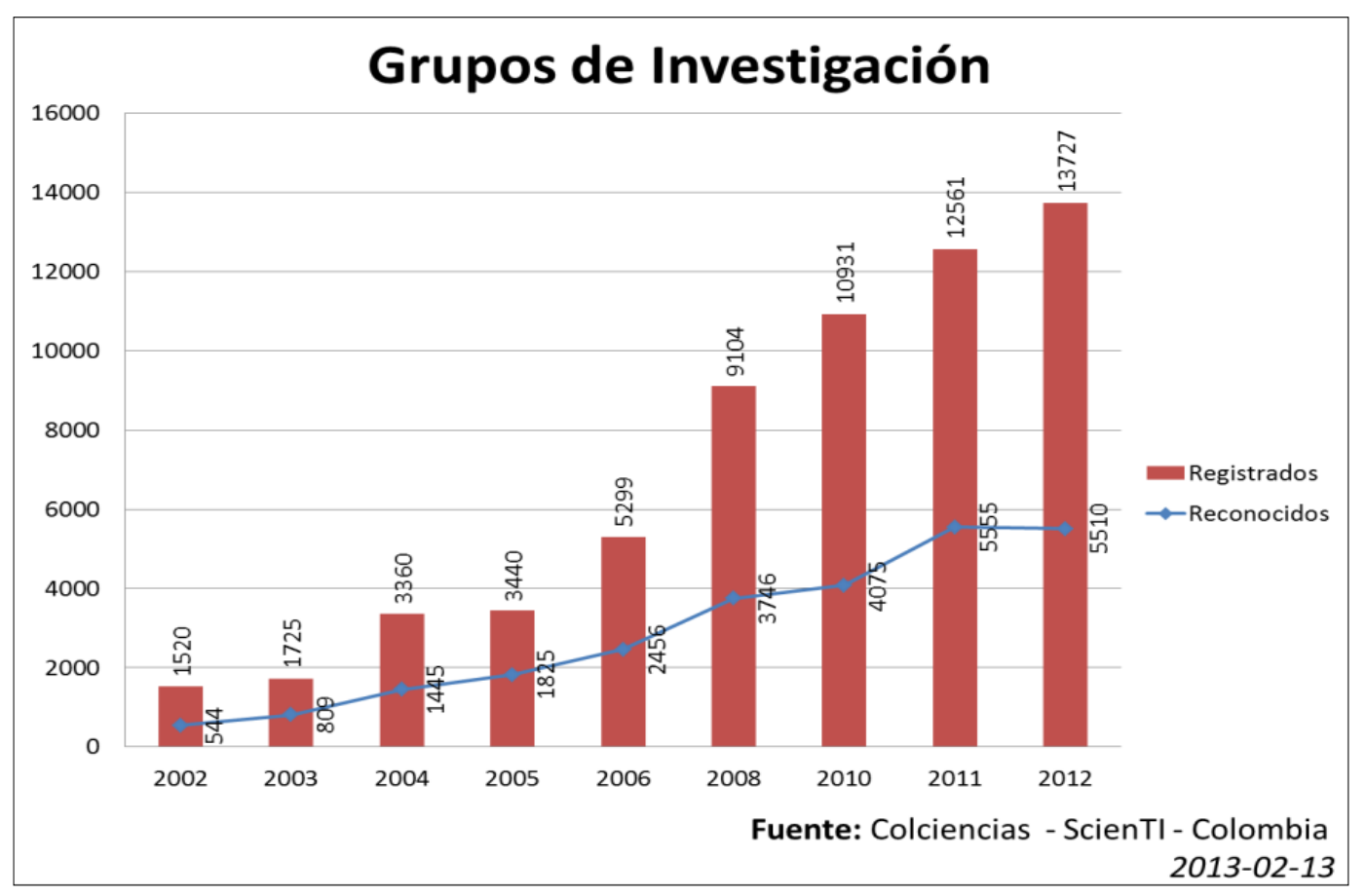

Figura 1. Reconocimiento Grupos de Investigación, convocatoria Diciembre 2012. (Tomado de Informe ejecutivo Colciencias Febrero, 2013) 


\section{Razón \# 3. Mejoramiento en el nivel de desarrollo del país}

De igual manera la plataforma Publindex/IBN (Índice Bibliográfico Nacional) tiene bajo su responsabilidad la indización de las revistas científicas prensadas en Colombia y clasificarlas con base en su calidad científica teniendo en cuenta una serie de criterios muy precisos que involucran desde el número de años de existencia de la revista, pasando por el precepto de que al menos un $60 \%$ de su contenido lo constituyan artículos originales, hasta la exigencia más dura de todas; que del número de Doctores que participan como miembros activos y evaluadores inscritos en su comité editorial, al menos 50\% de ellos demuestren su domicilio internacional. Publindex arranca su clasificación a partir de la categoría A1 (Revistas de mayor solidez en términos científicos y epidemiológicos) hasta la categoría $C$ (Revistas de menor rigor científico), estando en el intermedio las categorías A2 y B. De las 467 indizadas a nivel nacional en todos los campos de la investigación, 43 están exclusivamente relacionadas con la medicina. Desde el año 2011, 4 revistas están en categoría A1 (Revista de Salud Pública de la Universidad Nacional, Biomédica, Colombia Médica y la Revista Colombiana de Psiquiatría) obteniendo las 3 primeras su merecidísimo premio de estar referenciadas no solo en las más importantes bases de datos en Latinoamérica como Scielo, LiLACS, BIREME, Redylac sino además de estar reconocidas en las ligas mayores de la investigación a nivel mundial, como son Medline y Pubmed. En categoría A2 se encuentran 14, en B hay 12 y en $C$ nuevamente 14 . No obstante resulta un tanto paradójico que un país que tiene casi que una revista médica por cada millón de habitantes solamente haya alcanzado la indización en PubMed de un paupérrimo 6\% del total de sus publicaciones (Colciencias, Publindex, 2013).

Pero, no solo se trata de mejorar la figuración en el rankeo científico internacional sino en que las publicaciones se reflejen verdaderamente en mejores políticas de salud para ese mismo país, en que sus indicadores de salud mejoren ostensiblemente, en que la calidad de vida de sus habitantes sea la mejor posible especialmente después de los 65 años y que las estrategias de prevención realmente funcionen, se comporten como costo-efectivas y estén a disposición de la mayor cantidad de población posible. Tomemos el caso de los Estados Unidos, reconociendo, claro está que se trata de un país perteneciente al primer mundo. La investigación biomédica (que involucra cualquier disciplina relacionada con el campo de las ciencias de la salud incluyendo a la psicología) está financiada a partir de cuatro patrocinadores principales: recursos del gobierno federal, recursos provenientes del gobierno estatal, asociaciones privadas sin ánimo de lucro y la industria farmacéutica. Para el año 2007 estos recursos sumaron la astronómica suma de 101.1 billones de dólares, recursos que comparados con los asignados durante el año 2003 se incrementaron en un 14\%. En este punto resulta curioso como la situación en particular para un país como Colombia las cosas funcionan exactamente al revés, con recortes presupuestales entre un año y otro cercanos al $30 \%$. Con este presupuesto se logró en los Estados Unidos para el año 2008, aprobar 17 nuevas moléculas, 3 nuevas indicaciones para biológicos y la aprobación de 25 nuevos dispositivos médicos y aunque en cantidad, estas cifras puedan parecer insignificantes frente a las interminables dolencias que aquejan a la humanidad, se calcula que podrán reducir el impacto económico hasta en un $20 \%$ de la carga de enfermedad que encierran las enfermedades crónicas (Dorsey et Al., 2010)

Resulta más que probable que con un presupuesto de 380 mil millones como el asignado a Colciencias en 2013 no cambiará en mucho la perspectiva sanitaria de nuestro país y mucho menos estaremos en capacidad de sintetizar nuevos medicamentos o de probar mejores tecnologías, pero lo que si se constituiría en benéfico para la investigación médica en un país como Colombia es la consecución de nuevos recursos a partir de la construcción de alianzas tecnológicas privadas gubernamentales que beneficien por igual a las 2 partes involucradas y por sobre todo a la sociedad misma, así mismo estimular y facilitar los estudios colaborativos con otros países en donde la participación de la Nación sea especialmente con recurso fundamentado en el talento humano y la población a observar más que soportado en lo meramente económico.

\section{Razón \# 4. Referenciación}

Este es otro argumento que debe mover a los jóvenes investigadores a prensar sus observaciones y conclusiones, en cuanto a que si no las publican, sencillamente sus investigaciones no existen, y de refilón ayudarán a empujar a sus propias revistas para mejorar sus contenidos, optimizar su categorización y hacerlas más visibles a la comunidad científica internacional con la consecuencia de mejorar sus posibilidades de estar mejor indizadas. Esta condición conocida como referenciación es una de las más apetecidas por los mejores investigadores a nivel mundial, no solo porque beneficia al propio investigador y su equipo de trabajo, al volverlos más visibles sino también porque atrae mayor can- 
tidad de recursos o becas (grants) para financiación de sus nuevos proyectos de investigación. La referenciación facilita además que el investigador y su equipo de trabajo tengan mayor disponibilidad para difundir su trabajo en escenarios internacionales y de paso suceda en una forma más natural y eficaz, la estructuración de trabajos colaborativos (multicéntricos como les denominamos los epidemiólogos) en los que se reparten equitativamente los costos, los esfuerzos de los investigadores, se mejora ostensiblemente la confiablidad del estudio y se fortalece la imagen del investigador. Sin embargo no hay duda en que para lograr la referenciación se requiere de una inquebrantable cultura de investigación.

Hace poco más de 10 años, Gutierrez y Mayca en 2003, aprovecharon una reunión académica de jóvenes investigadores (estudiantes de medicina) con motivo del V Encuentro Internacional de Revistas Científicas Estudiantiles de Medicina para indagar acerca de las razones por las cuales a nuestros estudiantes les gustaba investigar. Antes de recapitular las razones que los motivaban, los directores del encuentro preguntaron cuántos de ellos habían publicado sus investigaciones; las estadísticas no pudieron resultar más que desalentadoras. Apenas el $27 \%$ logró culminar su trabajo de investigación y presentarlo en algún congreso, sin que ni uno solo se animara a publicarlo. Más caótico aún resulto el hecho de que casi el 50\% de los estudiantes Latinoamericanos encuestados asistentes al evento respondieron que no se sentían motivados para participar en proyectos de investigación. Por supuesto que aunque se trata de un tipo de estudiante en nivel de pregrado en los que alcanzar sus competencias asistenciales como estudiantes de pregrado se constituye en el requisito por excelencia para obtener su grado como médicos generales, la situación infortunadamente no es muy diferente a nivel de postgrado de primera y segunda especialización en donde la tasa de publicación, por lo menos en nuestro Hospital y nuestra Universidad no alcanza el 10\%. (Subdirección Docencia e Investigación Científica, Hospital Militar Central, 2012). No obstante los factores que identificaron a la hora de motivarse para investigar son, por defecto, exactamente los mismos que mueven a cualquier investigador: Generación de criterio (construcción y difusión de conocimiento), valoración personal (autoestima), carta de presentación (referenciación), feedback (estudios colaborativos).

\section{Razón \# 5. Perfeccionamiento en el ejercicio de la Medicina}

De igual manera investigar también ayuda a estructurar en forma eficiente el ejercicio cotidiano de la medicina. Constantemente los médicos al enfrentarnos a una condición clínica de un paciente repetimos cotidianamente un mini proceso de investigación, partimos con una entrada de datos (historia clínica), generamos hipótesis (formulación de diagnósticos diferenciales), analizamos (factores causales) planteamos un diagnóstico final (resultados) y generamos las mejores recomendaciones (conclusiones). El método científico practicado por los médicos con cotidianeidad, garantiza en últimas que las decisiones tomadas sobre los pacientes están cimentadas en recomendaciones (evidencias) que han pasado por juiciosos análisis estadísticos y epidemiológicos. En otras palabras investigar, perfecciona el tipo de medicina que estamos brindando a diario a nuestros pacientes. Adicionalmente la adquisición progresiva de habilidades obtenida desde la investigación, hace que se tengan herramientas metódicamente más objetivas para analizar con visión crítica los resultados y conclusiones de otros, no importa que sean contradictorias a las propias y que se disponga de la mejor autonomía para decidir qué conductas tiene un mejor posicionamiento en la práctica clínica cotidiana. En síntesis la investigación hace al ejercicio de la medicina un procedimiento mucho más técnico y menos riesgoso, pero al unísono mucho más participativo, analítico y prolijo a mejores resultados.

\section{Razón \# 6: Mejoramiento de su condición laboral}

Quizás lo más crítico en el proceso de investigación sea los beneficios tangibles que el profesional obtiene para sí mismo. Hasta ahora hemos hecho un resumen pormenorizado de los innumerables beneficios que la investigación brinda, como es el deber ser, en primera instancia a su paciente, en segundo lugar a su institución y en últimas, aunque suene descaradamente patriótico, a su país. Pero, ¿qué hay de los beneficios que le representan a él mismo? Definitivamente en países como el nuestro estos no son muy fáciles de encontrar. Sin embargo algunas Universidades en Colombia ya han empezado a discriminar en sus procesos de admisión aquellos candidatos a quienes les interese realmente este cuento de la investigación. Con mayor frecuencia se repara al interior de su hoja de vida hasta donde ha llegado en términos de investigación y existen algunas instituciones que otorgan un puntaje adicional, durante el proceso de admisión, a aquel candidato que pueda reportar el mayor número de trabajos publicados. En el mismo sentido algunas Universidades (Universidad Nacional, Universidad de la Sabana, Universidad del Rosario y Tecnológica de Pereira, por citar ejemplos) han empezado a seleccionar sus docentes mediante procesos que no solo involucran sus 
perfiles profesionales en términos de competencias sobre el binomio enseñanza-aprendizaje sino además le dan un valor preponderante a la producción científica que tenga el candidato. Esta discriminación resulta especialmente relevante al momento de seleccionar el mejor perfil, pero sobretodo al intentar seleccionar a un docente involucrado en los intríngulis de la investigación y que esté en disposición permanente de estimularla sobre sus discentes. Esta misma metodología se está aplicando para la selección de candidatos a posiciones especialmente en cargos directivos $\mathrm{u}$ operativos estrechamente relacionados con el quehacer de la docencia y la investigación (SEPAP 2011). En resumen, al menos para algunas instituciones, quizá las más aventajadas, la posibilidad de aspirar a una posición laboral dependerá en alguna medida de la madera y productos que el candidato tenga como investigador.

\section{Razón \# 7. Retribución económica}

Otra estrategia para motivar la investigación apunta a incentivos económicos directos entregados a la institución a la que representa el investigador o al investigador mismo y su equipo de trabajo. Aunque hoy en día son pocas las instituciones hospitalarias o Universitarias en Colombia que pueden darse el lujo de mejorar, los de por si lánguidos, ingresos laborales de sus empleados, han apostado a que cada publicación indizada o cada esfuerzo de transferencia de conocimiento, como por ejemplo la editorialización o la colaboración en capítulos de un libro, se transformen en puntos acumulativos que mejoran el nivel salarial del investigador en forma inmediata o al recibir su jubilación. Por supuesto estas sumas irrisorias nunca compensarán en la medida de lo justo lo que significan su esfuerzo y dedicación. En forma similar, aunque con una estrategia diferente, otros hospitales y centros de investigación están reconociendo, desde hace muy poco, paquetes económicos a cada publicación indizada que logre prensar el autor con su equipo de trabajo. En palabras sencillas el grupo investigador recibe cierta suma de dinero, generalmente entre 2 y 3 salarios mínimos legales vigentes por cada investigación que logre prensar preferiblemente en revistas médicas de alto impacto. En teoría los recursos obtenidos deberían destinarse a manera de financiación para iniciar un nuevo proyecto aunque esto pasa a ser de estricta autonomía del autor o autores del manuscrito. $\mathrm{Si}$ bien la estrategia puede resultar exitosa bajo el argumento de que a todos nos gusta que nos paguen por realizar bien nuestro trabajo, la comercialización de la investigación y el desarrollo de protocolos que solo atiendan a satisfacer estos ímpetus económicos puede hacer que su calidad se vulnere y se distorsione por completo la verdadera esencia de la investigación que es la de entregar la menor incertidumbre al paciente.

\section{Reflexiones finales}

Es claro que la prosperidad de un país no solo puede ser medida desde los ángulos que atañen exclusivamente a lo saludable de su economía, en el concepto están implícitos el nivel de bienestar, recreación y salud que sus colectividades dispongan. Una politica racional y eficaz de salud conlleva no solo a seleccionar las mejores opciones posibles con el fin de fortalecer sus politicas sanitarias, sino además a la construcción de novo de soluciones que atiendan a sus propias necesidades insatisfechas y que se aniden en las respuestas a situaciones de conflicto engendradas a partir de su entramado social. Es por esto que la investigación en salud resulta fundamental al momento de cuantificar que tan bien va un país y de paso que tan sólido resulta su entorno educativo, entre otras en cuanto a que el binomio educación-investigación no puede acontecer desarticulado, sus consecuencias deben apuntar siempre hacia la misma dirección y sus naturalezas deben permanecer estrechamente compartidas. No hay duda que investigar en salud hoy con respecto hace 20 años es, al menos, discretamente más reconfortante. La cantidad de científicos formados en investigación ha crecido ostensiblemente, aunque nunca en el número necesario para identificarnos como un país de naturaleza sólida investigativa, pero si existen evidencias que denotan esfuerzos mayormente individuales que gubernamentales para corroborar esta tendencia. Más recientemente el Gobierno ha intentado organizar con algún éxito grupos de investigadores discriminados por fortalezas académicas y administrativas apuntándole al fortalecimiento de líneas de investigación y fomentando su producción científica. El hecho de que existan acuerdos gubernamentales apuntando por lo menos a las buenas intenciones de destinar hasta el 10\% de las regalías Departamentales para financiar proyectos de investigación podría constituirse, de no politizarse ni viciarse con los manoseos de la corrupción, en un esfuerzo eficaz y regional para promover e impulsar la investigación a mayor escala. Necesariamente las regiones más aventajadas en los menesteres del quehacer investigativo en Colombia como lo son Antioquia, Valle, Santander y el Distrito Capital deberán solidarizarse con regiones de menor tradición investigadora y asesorarles y asistirles en el propósito de no desistir a los retos que le impondrá, en términos de investigación, esta nueva perspectiva. 
De igual manera, resulta menester abaratar los costos de investigación, una modificación favorable sobre este obstáculo, por increíble que parezca, también estimula en forma directa a los investigadores, y la estrategia desde nuestra perspectiva, radica en compartir conocimiento entre núcleos de investigadores, facilitar el acceso a las redes internacionales de información, implantar la sistematización del proceso, estimular la conformación de bases de datos a nivel Nacional e involucrar a otro tipo de profesionales que simplifiquen y racionalicen en términos de costos, los procesos subyacentes a la investigación. Finalmente el reconocimiento digno en términos de remuneración, estímulos, reconocimientos y la garantía de un proceso de formación continua en investigación para investigadores, desde los inicios mismos de su formación como médicos, con extensión a los cuatro puntos de la geografía nacional convertirá a Colombia en un término de 10 años o menos en un país ahora si, competitivo a la hora de investigar.

Conflictos de interés: $\mathrm{El}$ autor declara no tener conflicto de interés en la realización de este trabajo.

\section{Financiación:}

Financiación particular del investigador

\section{Referencias}

1. Capítulo II. De los derechos sociales, económicos y culturales. Artículo 44, 49, 67 a 71. Constitución Política de Colombia. 1991.

2. Lewison G. Beneficios de la investigación médica para la sociedad. Clínica Médica. 2005;131(5): 42-47.
3. Pedraza AM, Rodríguez-Martínez C, Acuña Cordero R. Validación inicial de una escala para medir el nivel de sobrecarga de padres/cuidadores de niños asmáticos. Biomédica. 2013; 33:361- 369

4. Proyecto Tuning Latinoamérica. 2011-2013. Innovación Educativa y Social. (Internet). Citado Marzo 27, 2013. Disponible en http: www.tuningal.org/es/publicaciones/cat_view/40-tuning-al-2004-2007

5. Pinilla A. Modelos pedagógicos y formación de profesionales en salud. Acta Médica Colombiana. 2011;36(4): 204-218

6. Banco Mundial. Gasto en investigación y desarrollo (Internet). Citado Marzo 28, 2013. Disponible en http://www.datos.bancomundial.org/indicador/GB.XPD.RSDV.GD.ZS

7. SCImago. (2007). SJR - SCImago Journal \& Country Rank (Internet). Citado Marzo 27, 2013. Disponible en http://www. scimagojr.com

8. Lang T, Siribaddana S. Clinical Trials Have Gone Global: Is This a Good Thing? PLoSMed. 2012;9(6): e1001228.

9. Patsopoulus N. A pragmatic view of pragmatic trials, dialogues. Clin Neurosci. 2011;13: 217-224.

10. Fog L. Colciencias se habría quedado sin cerebro. El Espectador. 2012 Septiembre 12

11. Colciencias. Informe ejecutivo de la Convocatoria Nacional para la definición de Grupos de Investigación, Tecnológica o de Innovación. 2012. Disponible en http:// Colciencias.gov. $\mathrm{co} /$ sites/default/files/ckeditor

12. Subdirección de Docencia e investigación Científica - Hospital Militar Central. Bogotá, Colombia. Informe de gestión. 2012.

13. Ministerio de Hacienda. Presentación de presupuesto Nacional, 2013. Disponible en http:www.minhacienda.gov.co/sites/ default/files/presupuestonacional.com

14. Ray E, Roulet J, Thompson J, Reminck J, Thai A, White Stellato $\mathrm{Z}$, et al. Funding of US Biomedical Research. JAMA. 2010;303(2): 137-143

15. Gutierrez C, Mayta P. Publicación desde el Pre Grado en Latinoamérica: importancia, limitaciones y alternativas de solución. CIMEL. 2003;8(1): 54-60

16. Sociedad Española de Pediatría Extrahospitalaria y Atención Primaria (SEPAP). Manual de iniciación de la Investigación en pediatría de atención primaria. 2011 